\title{
Kritische Kartographie, kritische Praxis und kritisches Kartieren. Mündige geographische Bildung mit digitalen Karten und Geodaten
}

\author{
" boris.michel@geo.uni-halle.de, Institut für Geowissenschaften und Geographie, Martin-Luther-Universität Halle-Wittenberg ( $\triangle$ korresp. Autor) \\ **inga.gryl@uni-due.de, Institut für Geographie \& Institut für Sachunterricht, Universität Duisburg-Essen
}

eingereicht am: 21.05.2021, akzeptiert am: 17.09.2021

\begin{abstract}
Der Beitrag stellt dar, wie basierend auf fachwissenschaftlicher Kritischer Kartographie einerseits und Kartieren als kritische Praxis andererseits fachdidaktische Herangehensweisen an kritisches Kartieren in geographischer Bildung entwickelt wurden. Es werden entsprechende unterrichtliche Anwendungsbeispiele vorgestellt, die insbesondere Einblicke dahingehend erlauben, wie kritische und mündige Zugänge auch in einer digital gestützten Kartierungspraxis möglich sind.
\end{abstract}

Keywords: Kritische Kartographie, Kritische Praxis, Counter Mapping, Reflexive Kartenarbeit, Digitalisierung

\section{Critical cartography, critical practice, and critical mapping. Mature geography education through digital maps and geodata}

This paper explains how didactical approaches for critical mapping in geography education have emerged from a subject perspective of critical cartography on the one hand and a critical practice on the other hand. It presents related practical examples that particularly provide insights regarding the opportunities for critical and mature approaches within a digital mapping practice.

Keywords: critical cartography, critical practice, counter mapping, reflexive mapping, digitalization

\section{$1 \quad$ Einleitung}

Karten gelten zweifelsohne als „die“ klassische Form der Visualisierung geographischer Sachverhalte und als „die“ geographische Methode. Carl Sauer beschreibt Karten in seinem geographiedidaktischen Klassiker „Education of a Geographer“ 1956 als die „language of geography“ und er proklamiert „liking maps and thinking by means of them" sei eine wesentliche Eigenschaft von Geograph*innen. „Show me a geographer who does not need them constantly and want them about him, and I shall have my doubts as to whether he [or she] has made the right choice of life“ (Sauer 1956: 289). Diesem Selbstverständnis entsprechend, gehören Kartieren und die Grundlagen der Kartographie zu den wesentlichen Bestandteilen geographischer Ausbildung und Lehre. War die Karte mindestens bis in die 1980er-Jahre „das“ Ausdrucksmedium der Geographie und proklamierte die traditionelle Geographie, dass ein Thema, welches nicht zu kartieren sei, vermutlich kein legitimes Thema der
Geographie sei, so ist diese Gewissheit seither durch eine Reihe von Kritiken erschüttert worden. Die sozial- und kulturgeographische Forschung hatte in den letzten Jahren vielfach ein eher ambivalentes Verhältnis zu Karten.

Einen wesentlichen Beitrag zu dieser Verschiebung hat eine Debatte beigetragen, die seit den späten 1980er-Jahren unter dem Label der critical cartography firmiert. Der Begriff der „Kritischen Kartographie“ bezeichnet eine Auseinandersetzung mit den sozialen und politischen Dimensionen von Karten, Kartieren und Kartographie und umfasst eine Doppelbewegung aus theoretischer Kritik und kritischer Praxis (Crampton \& Krygier 2005). Während Kritische Kartographie in erster Linie im akademischen Feld angesiedelt ist und eine Kritik der a-politischen, a-historischen, eurozentrischen Perspektive auf Karten, Kartieren und Kartographie formuliert, beschreibt kritisches Kartieren ein heterogenes Feld politischer, aktivistischer und künstlerischer Praktiken des Kartenmachens, des Kartierens und des Kartennutzens und positioniert sich 
jenseits der etablierten Kartographie und oftmals in direkter Kritik an dieser. Beide Dimensionen überschneiden sich an vielen Stellen, beiden geht es um eine Kritik hegemonialer Erzählungen in und durch Karten sowie um eine kritische Auseinandersetzung mit dem Machen und der „Macht der Karten“ (Wood 1992), und beide lassen sich in kritisches Kartieren in schulischen Kontexten übertragen.

In diesem Beitrag sollen beide Dimensionen kurz vorgestellt werden, bevor wir Überlegungen zu einer schuldidaktischen Übersetzung formulieren.

\section{Theoretische Grundlagen Kritischer Kar- tographie}

\subsection{Kritische Kartographie - Kritik kartographi- scher Diskurse und kartographischer Praxis}

Indem sie die seit den 1960er-Jahren in der Kartographie diskutierten semiotischen und kommunikationstheoretischen Zugänge zu Karten mit poststrukturalistischen und postmodernen Autor*innen und Debatten verbanden, nahmen kritische Kartograph*innen Karten und Kartographie als Teil machtvoller Zeichensysteme, sozialer Texte, Diskurse und Ideologien in den Blick. Wegweisend und paradigmatisch stehen hierfür Arbeiten wie Brian Harleys „Deconstructing the Map“ (Harley 1989) und Denis Woods „The Power of Maps” (Wood 1992). Diese Texte zielten darauf ab, Karten und Kartographie ihren Anschein wissenschaftlicher Neutralität und Objektivität zu nehmen und machten diese damit selbst zu Objekten kritischer Analyse. Aus dieser Perspektive lag die Aufgabe einer kulturtheoretisch informierten und kritischen Perspektive in der Dekonstruktion, Dekodierung oder Ideologiekritik von Karten und Kartographie. Es galt folglich, die diskursiven Regeln und die Rhetorik kartographischer Wahrheitsproduktion herauszuarbeiten und Karten als Ausdruck und Verstärker gesellschaftlicher Machtverhältnisse zu begreifen. Diese Autor*innen problematisierten die Karte als visuelles Zeichensystem, das in hohem Maße eine Sedimentierung und Fixierung, eine Verdinglichung und Verräumlichung dynamischer sozialer Verhältnisse sowie ihre Übersetzung in starre räumliche Muster nahelege. Indem Karten und Kartographie als (soziale) Texte, Diskurse und Zeichen gelesen wurden, wurde es möglich, sie als machtvolle und produktive Praktiken der Erzeugung von Welt und Weltverhältnissen zu verstehen.

Der Selbsterzählung der Kartographie als universeller und objektiver Wissenschaft der mathematisch korrekten verkleinerten und geebneten Abbildung räumlicher Beziehungen von Dingen auf der Erd- oberfläche stellen kritische Kartograph*innen eine Beschreibung der Disziplin als ein Instrument der Erzeugung staatlichen Territoriums und Reproduktion sozialer Ordnung gegenüber, das aufs Engste mit der eurozentrischen Geschichte der modernen Wissenschaften, den europäischen Nationalstaaten und dem europäischen Kolonialismus verbunden ist. Seien es die großen kartographischen Landaufnahmen in Europa seit dem 17. Jahrhundert (Branch 2015) oder die kartographische Erschließung außereuropäischer Kolonien (Edney 1997; Akerman 2009), die nicht nur die militärische, politische und ökonomische Kontrolle ermöglichte, sondern immer auch mit der Unsichtbarmachung indigener Ansprüche und Räume einherging, welche oftmals eben nicht in der räumlichen Logik europäischer Territorialstaaten und europäischer Kartographie formuliert waren. Ein solches kartographisches Wissen wurde instrumentell für die Herstellung von Containerräumen und räumlichen Wissensspeichern einer Welt, die zunehmend containerräumlich organisiert ist - von einem modernen Kataster und Bodenmarkt über multi-skalare Verwaltungsregime bis zu einer Weltordnung souveräner territorialer Nationalstaaten und der Konstruktion der Welt als einer Einheit, wie sie etwa in den ersten Fotographien und Satellitenbildern in den 1960er-Jahren zum Ausdruck kommt (Cosgrove 2001; Pickles 2004).

Was Karten auszeichnet, ist aber nicht nur ihre historische Funktion, staatliches Territorium zu beanspruchen und sichtbar zu machen, sondern auch das, was James Scott (Scott 1998) als "Lesbarmachung“ bezeichnete. Diese war und ist eine wesentliche Voraussetzung biopolitischer und governmentalisierter Staatlichkeit (Elden 2007; Hannah 2000). Seit Mitte des 19. Jahrhunderts wurden thematische Karten zu einem wichtigen Instrument, um das rapide anwachsende statistische Wissen über Staat und Bevölkerung durch Visualisierung und Verräumlichung verstehbar und damit als Machtwissen produktiv zu machen. Karten verleihen der sozialen Frage eine graphische Repräsentation und machen sie damit als räumliches Problem adressierbar. Die mit ihnen verbundenen Momente der Verräumlichung sozialer Phänomene, Abstraktion, Fixierung und Subjektanrufung sind auch für heutige Kartierungen sozialer Sachverhalte relevant. Neben stark text- und diskursorientierten Ansätzen der kritischen Kartographie haben sich wie in vielen Teilen der Kultur- und Sozialgeographie - seit den frühen 2000er-Jahren zudem Ansätze in der Kartographie etabliert, die stärker den praktischen und prozessualen Charakter von Karten und Kartieren betonen (Edney 2019; Dodge et al. 2009). $\mathrm{Zu}$ dieser Wende von Text hin zu Praxis haben nicht zuletzt die radikalen Veränderungen von Karten und 
Kartographie im Zuge der Digitalisierung und die Entstehung offener und leicht anwendbaren OnlineKarten beigetragen. Karten zu machen, bedarf heute keiner kartographischen Ausbildung mehr.

Als Forschungsmethode zielt ein solcher Zugang daher sowohl auf eine kritische Analyse kartographischer Darstellungen als auch ihrer Entstehungsprozesse ab. Neben den oben genannten Beispielen fallen darunter u.a. Arbeiten zu Karten geopolitischer Diskurse in der Wissenschaft oder dem Einsatz von Kriminialitätskartierungen in Diskursen städtischer Sicherheitspolitik (Belina 2007) ebenso wie Auseinandersetzungen mit den sozialen Dimensionen kollaborativer Online-Kartierung oder den Raumproduktionen sozialer Medien.

\subsection{Jenseits dieser Kritik - Kritisches Kartieren als kritische Praxis}

Kritische Kartographie hat im oben beschriebenen Sinne zu einer Skepsis gegenüber Karten und Kartographie in der Kultur- und Sozialgeographie beigetragen. Aus Perspektive dieser Kritik wurden Karten von einem Mittel der Darstellung und Erzeugung geographischen Wissens zu einem Objekt, das selbst Gegenstand kritischer Analyse wurde. Diese frühe Kritische Kartographie war stark von den Diskurs- und Machtbegriffen Michel Foucaults (Foucault 2000) inspiriert und betonte dabei sowohl die repressiven als auch die produktiven Momente kartographischen Machtwissens. Dennoch blieb diese Perspektive meist eine, die der Macht des Staates, von Unternehmen oder den Wissenschaften ein Primat zusprach und nicht den mannigfaltigen Widerständigkeiten und Subversionen. Im folgenden Abschnitt geht es um ein Verständnis von und Verhältnis zu Karten, das gerade das Offene und Subversive der Karte betont und das auch in der deutschsprachigen Geographie sich eines wachsenden Interesses erfreut (Michel 2017; 2021).

Wenn die oben formulierte Kritik in erster Linie aus dem akademischen Feld von Kartographie, Critical GIS und Geographie stammt, so hatten alternative Ansätze einer kritischen Praxis des Kartierens ihren Ausgangspunkt oftmals außerhalb des akademischen Felds und zumeist explizit in Kritik der mit diesem verbundenen Rationalitäten. Zwei Perspektiven sind dabei zentral:

Einerseits gibt es eine in den 1960er-Jahren einsetzende Tradition des Counter-Mappings, die dem hegemonialen kartographischen Wissen andere Karten gegenüberstellt, sei es in Form indigener Counter-Mappings in Konflikten um die Anerkennung von Landrechten in ehemaligen Kolonien wie z. B. Kanada (Bryan \& Wood 2015; Sletto et al. 2020) oder in Form kritischer Interventionen in einem von Rassismus, Armut und Bildungsungleichheit gepräg- ten städtischen Raum, wie die Arbeiten der Detroit Geographical Expedition rund um William Bunge und Gwendolyn Warren (Bunge 1971; Warren et al. 2019; Katz 2021). Diese frühen Arbeiten öffneten die Karte für neue Akteur*innen, neue Inhalte und neue kartographische Praktiken in aktivistischem und künstlerischem Bereich. Besonders deutlich wird dies am Beispiel kollaborativer und partizipativer Kartierungen indigener Gruppen, bei denen marginalisierte Akteur*innen mittels kollektiver Praktiken des Kartierens sowohl eigene territoriale Ansprüche als auch Weltverständnisse formulieren und kommunizieren. Dabei finden diese Praktiken vielfach in einem Spannungsverhältnis zwischen den Konventionen und Technologien der etablierten Kartographie und ihrer Autorität auf der einen Seite sowie „unprofessionellen" Visualisierungen auf der anderen Seite statt. Andererseits nutzten zahlreiche Künstler*innen Karten und eigneten sich diese an, verwendeten Karten als Kunst und machten Kunst mit Karten, vielfach verbunden mit einer radikalen Kunst- und Gesellschaftskritik. Von der ironischen Verwendung einer gänzlich leeren Karten in Lewis Carrolls „The Hunting of the Snark" oder satirische Imaginationen die kartographische Darstellungen und deren wissenschaftliche Exaktheitsproklamation ins Absurde überzeichnen, über die psychogeographischen Kartenexperimente und Explorationen der Situationistischen Internationale bis zu Öyvind Fahlströms Sketch for World Map Part von 1972 fanden Künstler*innen in Karten ein probates Mittel, alternative Formen der Welt- und Raumerfahrung zu bearbeiten und sich an der Wahrheits- und Sichtbarkeitsbehauptung von Karten abzuarbeiten (Wood 2010; O’Rourke 2016; Edney 2019).

Kritisches Kartieren (Mogel \& Bhagat 2008; Orangotango+ 2018) kann vor diesem Hintergrund als eine vielschichtige Praxis beschrieben werden, die andere Inhalte visualisiert und damit eine Herausforderung an hegemoniale kartographische Wissens- und Raumproduktionen formuliert. Kritisches Kartieren öffnet das etablierte und professionalisierte Feld der Kartographie für andere Akteur*innen, sei es in Form von partizipativem Kartieren durch marginalisierte Gruppen, die hiermit eine kartographische Stimme erhalten, dem Feld von Public Participation Geographic Information System oder auch neuen Formen kollaborativer Onlinekarten (Duggan 2019; Bittner $\&$ Michel 2018). All dies ist oft verbunden mit Praktiken und einer Ästhetik, die sich gegen die hegemonialen Formen richtet, und vielfach mit einer reflexiven Perspektive, die den konstruierten Charakter und die Entscheidungen kartographischer Rationalität und Positionalität offenlegt und damit zu einer produktiven Destabilisierung der Karte als Medium der Raumkonstruktion beiträgt (Halder \& Michel 2018). 


\section{Praxisbeispiele Kritische Kartographie}

\subsection{Didaktische Ansätze kritischer Kartographie und kritischen Kartierens}

Schulische Kartenarbeit war lange Zeit durch eine relativ unkritische und formalistische Herangehensweise geprägt (vgl. Rinschede 2003) und später durch eine methodisch nicht weiter ausgeführte Reflexion (vgl. Hemmer et al. 2010). Eine kritische schulische Kartenarbeit ist inspiriert durch beide bereits dargestellte Dimensionen: Der akademische Diskurs ist in der theoretisch aufgeladenen Konzeptionalisierung mit Foki auf Dekonstruktion und Digitalisierung dominant, und die kritische Praxis - künstlerische wie identitätsbildende Zugänge aufgreifend - dürfte inspirierend sein hinsichtlich der schulischen Implementation (vgl. Nöthen 2016; Hintermann et al. 2018).

Als direkte Adaption von Harleys Vorlage macht „Konstruktivistische Kartenkompetenz“ (Gryl 2009) den Anfang und wird ergänzt um „Reflexive Kartenarbeit" (Gryl 2012), die neben dem eher externalisierten Reflektieren des Mediums auch die Reflexion des eigenen Umgangs damit einbringt und empirisch nachweist. Dieser Grundsatz des Hinterfragens der eigenen Position ist essentiell für (schulische) kritische Kartenarbeit, da Karten letztlich immer intentional genutzt werden und Einfluss auf die Erkenntnisgewinnung sowie spätestens seit ihrer Omnipräsenz mit der Einführung von Geolocation Services auch für das alltägliche Handeln haben. Bezugnehmend auf ein plurales Verständnis von Raumkonzepten (Wardenga 2004) wurde zugleich die grundlegende Produktion von Räumen (vgl. handlungstheoretische Sozialgeographie, Werlen 1993, adaptiert z. B. in Jekel 2008) hinter und mit Kartenproduktion stärker in den Blick genommen. Dies verschafft durch den engeren Bezug zu Handlungsräumen ein Verständnis der Veränderlichkeit von in Karten reproduzierten Diskursen und das Potential, selbst Räume zu konstruieren, angeregt durch die Praxis des Counter Mappings (Turnbull 1998). Dies erfordert eine produktive Herangehensweise, die in den Schulen mit zunehmender Medienproduktion im Zuge der Kompetenzorientierung, aber auch durch einfach anwendbare digitale Tools vorangebracht wird - trotz der schulischen Tradition des Primats der Rezeption. Damit konnte auch aktives Kartieren jenseits der GIS-Bildung realisiert werden. Der Ansatz Spatial Citizenship (Gryl \& Jekel 2012) führt die Kompetenzanforderungen einer solchen Kartenproduktion mit emanzipatorischer Stoßrichtung (Vielhaber 2000) auf: Neben basalen technischen Fähigkeiten sind dekonstruierende wie reflexive Kompetenzen und inhaltliche Bezüge geographischer Bildung involvierend, sowie kommunikative Kompe- tenzen der Argumentation, der Aushandlung und der Partizipation notwendig.

Der Ansatz der viralen Raumproduktion (Kanwischer \& Schlottmann 2017) macht deutlich, welche Rolle dabei geomediale Web-Kommunikation spielt: Eine didaktische Berücksichtigung der Kultur der Digitalität (Stalder 2017), etwa nach Dorsch und Kanwischer (2019), hilft, die Schlagkraft von Digitalisierung bzgl. sozialer Implikationen in kritisches Kartieren einzubringen und damit die kritische Reflexion des (Web-)Kartierens zu aktualisieren.

\subsection{Beispiel: Kritisch analysieren und sich be- teiligen - die Wheelmap}

Die Wheelmap (wheelmap.org) ist ein Projekt, in dem durch Nutzer*innen und Freiwillige Orte mit und ohne Barrieren für Rollstuhlfahrer*innen kartiert werden. Der Verein Sozialhelden e.V. strebt damit an, mit Hilfe einer großen Community Personen im Rollstuhl die Planung ihrer Navigation durch Städte zu erleichtern, ihren Aktionsradius und ihre Selbstständigkeit zu vergrößern. Lediglich mit einem Open-Street-Map-Login und damit einer vergleichsweise geringen Preisgabe persönlicher Daten wählen Nutzer*innen Objektkategorien für einen Standort und bewerten ihn nach einem Ampelsystem. Freilich hat auch dieses System seine Grenzen, da etwa Treppen und Stufenhöhen, aber nicht schwere Türen und Ausmaße von Fahrstühlen in die Bewertung einbezogen werden.

Für den Unterricht ${ }^{1}$ eignet sich dieses Projekt insofern, als dass sehr einfach und datensicher durch Schüler*innen zur Karte beigetragen und damit selbst kartiert werden kann. Es besteht kein grafischer und kein inhaltlicher Gestaltungsspielraum, sondern es wird der Gestaltung und der sozialen Agenda der Initiator*innen gefolgt. Gleichwohl können Schüler*innen mit äußerst geringem technischem Aufwand durch Kartierung partizipieren. Zur Datenaufnahme würde sich eine gemeinsame Exkursion eignen (etwa im Sinne einer „Kampagne", wheelmap.org) oder individuelle Datenaufnahme auf alltäglichen Wegen. Neben der methodischen Ausrichtung können auch soziale Aspekte wie Inklusion sowie sozialgeographisch zentrale Felder wie „Partizipation im Stadtraum“ und „Recht auf Stadt" thematisiert werden.

Mit Kritischer Kartographie wird die Karte zudem selbst zum Gegenstand des zu Hinterfragenden. Zunächst einmal ist die Karte ein Beitrag zur

Die unterrichtliche Umsetzung wurde von Gryl und Atteneder für die Neuauflage der Bildungsstandards Geographie (DGfG 2020) konzipiert. 
UN-Behindertenrechtskonvention (2006), da sie die darin geforderte Inklusion vorantreibt. Anderseits ist sie damit auch die Übernahme von Aufgaben, die durch einen Staat ratifiziert und zugesagt wurden, durch Ehrenämtler und Betroffene im Sinne zivilgesellschaftlichen Engagements und Selbsthilfe. Dies zeugt von Verantwortungsbewusstsein, Solidarität und Eigeninitiative, aber auch von einem Versagen staatlicher Strukturen und einem Auslagern in kompensatorische Eigenverantwortung (Bröckling 2007; Bittner \& Michel 2018). Engagement wie dieses schafft neue Freiheiten, kann aber auch zur Konsolidierung defizitärer Strukturen beitragen, indem bei einem praktischen Vermittlungsinteresse (vgl. Vielhaber 2000) verblieben wird. Andererseits aber kann eine Karte voller roter Punkte auch als Mittel politischer Kommunikation genutzt werden, um die enormen Lücken im Erfüllen der UN-Konvention aufzuzeigen und politischen Druck zu erzeugen. Damit würde ein weiterer potentieller Nebeneffekt der Karte etwas geheilt werden: Rollstuhlfahrer*innen meiden mit gewisser Wahrscheinlichkeit rot markierte Orte und bleiben damit an diesen unsichtbar, weil absent, was den allgemein wahrgenommenen Bedarf nach Barrierefreiheit für diese Orte negativ beeinflusst. Die unterrichtliche Diskussion auf einer Metaebene, welche Chancen und Grenzen diese Karte birgt, und wie direkte Unterstützung mit politischem Einfluss verbunden werden kann, ist lohnend für die Vermittlung eines kritischen/reflexiven Zugangs zu Kartographie, Raumproduktion und Gesellschaft. Insbesondere das Potential und die Grenzen des Machtausgleichs durch Spatial Citizenship für entlang von Intersektionalitätslinien ausgegrenzte Gruppen wird interdisziplinär und lebensweltlich beleuchtet und trägt damit zu einer mündigkeitsorientierten Bildung (Dorsch \& Kanwischer 2019) und einer reflexiven Haltung bzgl. des Selbst-Gesellschafts-Verhältnisses bei.

\subsection{Beispiel: Tracking und Tracing verstehen}

Zahlreiche Applikationen für mobile Endgeräte sind auf Geolokalisierungsbezug über GPS, WLAN, Mobilfunk oder Bluetooth (letzteres für räumliche Lagebeziehungen der Nähe) angewiesen bzw. sind ohne sie nur eingeschränkt nutzbar, selbst wenn sie nicht in jedem Fall immanent für die angebotenen Funktionen ist oder Nutzer*innen konkret Karten angeboten werden. Vor dem Hintergrund einer kritischen Annäherung an Kartieren lohnt sich daher auch ein Blick auf mutmaßlich ungewolltes und auch unbewusstes Kartieren bzw. Kartiert-Werden. Es ist die Entscheidung zu treffen, auf Service und Teilhabe zu verzichten, oder aber dafür Daten freizugeben. Daher muss, obgleich die Vermittlung der technischen Grundlagen der Geolokalisierung in den Bereich der informatischen Grundbildung fällt, diese stets um die sozialen Implikationen der Verortung, etwa Spatial Privacy (Armstrong \& Ruggles 2005), ergänzt werden. Auch wenn die Verwertung von Standortdaten - mit Blick auf komplexe AGB - je nach Anwendung in unterschiedlichem Maße erfolgt, kann davon ausgegangen werden, dass im Falle einer Speicherung umfassende Informationen abgeleitet werden können. Gerade die Einwahl mit einem Login für mehrere Dienste erlaubt über das Sammeln von Standortdaten das Erstellen von Bewegungsprofilen zu einem*einer Nutzer*in, wobei auch bei anonymisierten Standortdaten eine Identifikation von Personen und Schlussfolgerungen über deren Gewohnheiten möglich sein kann (Datenschutz.org 2021). Medienwirksame Beispiele wie die genaue Lokalisierung von Militärstützpunkten durch mit Fitnessarmbändern joggende Armeeangehörigen (The Guardian 2017) sind nur ein Beispiel.

Eine einfache Übung zur Interpretation von Standortdaten ist das Tracking bzw. Tracing (Stark 2012), das zeitgleiche oder zeitversetze Nachverfolgen der Bewegungsdaten. Gesammelt mit einfachen Apps durch Schüler*innen können Mitschüler*innen vor dem Hintergrund von Orten, Zeiten und Verweildauern in Programmen wie Google Maps, die diesen Daten zugleich konkrete Informationen über besuchte Orte aus vielfältigen Quellen (Mashups) hinzufügen, Rückschlüsse über Aktivitäten ziehen. Zugleich erfahren Schüler*innen, wie schwer es ist, das Tracking/Tracing (ohne den ,Service' abzuschalten) zu überlisten, d. h. andere Standorte und Aktivitäten vorzutäuschen.

Hinzu kommt das offene Teilen von Standorten oder Tracks, das zwischen Authentizität (z. B. Bewertungen von besuchten Einrichtungen, Dokumentation von Routen), dem Herstellen von Identität über Distinktion und der Gemeinschaftlichkeit über physische Bezüge, und der Selbstexposition changiert. Eine Spielart ist das GPS-Drawing, bei dem der mit Lokalisierungsequipment versehene Körper zum Pinsel wird, der durch Ablaufen/-Fahren von Strecken Bilder als digitale Layer über die Landschaft legt. Es bietet sich an, basale Orientierungskompetenzen mit GPS-Drawing zu fördern (Gryl 2016) und dabei gleichzeitig ein Bewusstsein für die ubiquitäre Aufzeichnung von Positionen zu entwickeln. Beispielweise wird eine Figur in eine Karte eingezeichnet - wobei ein sinnvoller Maßstab und Distanzen zu beachten sind - und mit Hilfe der Karte abgelaufen, während in der Tasche der Track aufgezeichnet und später mit der Zeichnung und weiteren Hilfsmitteln (Street View etc.) verglichen wird. Andererseits stellt sich die Frage nach der gegenwärtigen und zukünftigen Relevanz einer solchen Orientierungskompetenz mit Karten, wenn ohnehin Orientierung über eine 
stets die Verortung sichtbar machende Navigationsapp erfolgen kann.

Im Sinne von "beyond the geotag" (Crampton et al. 2013) werden auch nicht georeferenzierte Spuren unbewusst und bewusst hinterlassen, etwa in Instagram durch ortsbezogene Hashtags. Neben Exposition und Spatial Privacy ist hier noch einmal mehr die Bedeutungszuweisung nicht nur durch die Konnotation des Ortes an die postende Person im Sinne der Inszenierung des Selbst, sondern auch durch die postende Person an Orte hoch relevant. Indem Hashtags in ihrer Formulierung oder Kombination raumbezogene Konnotationen tragen, können so Images von Orten (und Menschen) gesetzt und eventuell wirksam geteilt werden (Kanwischer \& Schlottmann 2017). Hashtags können im Unterricht Gegenstand des Hinterfragens und zugleich Medium der Konstruktion etwa von Counter-Bedeutungen und bilden - obgleich nicht zwangsläufig mit kartographischen Darstellungen verbunden - einen Zugang zum Kritischen Kartieren im weiteren Sinne.

\section{Diskussion der Methode aus fachwissen- schaftlicher und fachdidaktischer Pers- pektive}

GIS ist - weiterhin - eine Expert*innendomäne des Kartierens, die eine relativ hohe Komplexität der Datenbereitstellung und -analyse erlaubt und als Forschungsdomäne räumlicher Analyse bestehen bleibt. Allerdings etablieren sich daneben weitere Domänen, in denen Expertise sich über inhaltliche Bezüge und/ oder Kompetenzen in der Strategie der erfolgreichen Raumkommunikation definiert, und somit auch über neue Bezugsrahmen jenseits formaler Abschlüsse. Mit Referenzialität und Gemeinschaftlichkeit gegenwärtiger Webkultur sind Rezeption und Produktion von Kartierungen eng miteinander verflochten. Auch mit rein qualitativen Repräsentationen können auf diese Weise machtvolle Diskurse geschaffen werden, was die Frage nach der Gestaltung von Argumentation, Verantwortung, Wahrheitsgehalt von ohnehin konstruierten Bedeutungszuweisungen und ethischen Rahmungen Kritischen Kartierens aufwirft - aufbauend auf den diesbezüglichen Überlegungen aus Spatial Citizenship (Gryl et al. 2017).

$\mathrm{Zu}$ verzeichnen ist auch eine Verschiebung zu mehr Medienvielfalt, wie ortsbezogene Instagram-Posts zeigen. Angelehnt an die seit Langem existente künstlerische Praxis Kritischen Kartierens könnte so der Verarmung vieler kartographischer Darstellungen in Form von Dot Maps begegnet werden. Schule kann helfen, eine Sprache der Karten jenseits von Konventionen zu erkunden, etwa durch die Analyse künstlerischer
Kartierungen und deren eigener Umsetzung (Nöthen 2016; Dickel 2016) sowie phänomenologischer Herangehensweisen (Pettig 2019). Da Karten hypothesengeleitet genutzt werden (MacEachren 1994), regt diese jenseits von Konventionen potentiell deutungsoffene Vorgehensweise Betrachter*innen zur eigenen Weltaneignung an und kann zugleich helfen, durch den in künstlerischen Techniken involvierten Zufallscharakter Erkenntnisprozesse im Kartieren anzuregen und im Zuge der Repräsentation weitere Perspektiven auf Räume einzunehmen.

Ausblick nehmend bedarf es angesichts der wachsenden Komplexität in der geographischen Bildung eines durch Kritische Kartographie und Praxis inspirierten, aber eben auch neuen Blicks, der lebensweltliche Erfahrungen in Erkenntnisse überführt und der nach und nach theoretisch gefüllt wird. Jenseits der aufgezeigten Forschungsinstrumente von GIS liegt mit der gegenwärtigen Produktion von Geodaten durch Lai*innen ein Forschungsfeld vor, das ebenfalls zur Theoriebildung beitragen kann.

\subsection{Danksagung}

Dank an den Publikationsfonds der Martin-LutherUniversität Halle-Wittenberg für die Förderung der Publikation.

\section{Literatur}

Akerman, J. R. (Hrsg.) (2009): Cartography and the mastery of empire. University of Chicago Press, Chicago.

Armstrong, M. P. \& A. J. Ruggles (2005): Geographic information technologies and spatial privacy. In: Cartographica 40(4). S. 63-73. DOI: https://doi.org/10.3138/ RU65-81R3-0W75-8V21

Belina, B. (2007): Zur Kritik von Kriminalgeographie und Kriminalitätskartierung ... und warum deren heutige Bemühungen noch hinter Quetelet zurückfallen. In: Tzschaschel, S., H. Wild \& S. Lentz (Hrsg.): Visualisierung des Raumes. Karten machen - die Macht der Karten. IFL, Leipzig. S. 241-255.

Bittner, C. \& B. Michel (2018): Partizipatives Kartieren als Praxis einer kritischen Kartographie. In: Wintzer, J. (Hg.): Sozialraum erforschen: Qualitative Methoden in der Geographie. Springer, Wiesbaden. S. 297-312.

Branch, J. (2015): The cartographic state. Maps, territory and the origins of sovereignty. Cambridge University Press, Cambridge.

Bröckling, U. (2007): Das unternehmerische Selbst: Soziologie einer Subjektivierungsform. Suhrkamp, Frankfurt am Main.

Bryan, J. \& D. Wood (2015): Weaponizing maps. Indigenous peoples and counterinsurgency in the Americas. The Guilford Press, New York/London. 
Bunge, W. (1971): Fitzgerald, Geography of a Revolution. Schenkman, Cambridge.

Cosgrove, D. E. (2001): Apollo's Eye. A Cartographic Genealogy of the Earth in the Western Imagination. John Hopkins University Press, Baltimore.

Crampton, J. W., M. Graham, A. Poorthuis, T. Shelton, M. Stephens, M. W. Wilson \& M. Zook (2013): Beyond the geotag: Situating 'big data' and leveraging the potential of the Geoweb. In: Cartography and GIScience 40(2). S. 130-139. DOI: https://doi.org/10.1080/15230406.2 013.777137

Crampton, J. \& J. Krygier (2005): An Introduction to Critical Cartography. In: ACME: An International E-Journal for Critical Geographies 4(1). S. 11-33.

Datenschutz.org (2021): Standortdaten: Bequemes Feature oder Überwachung? https://www.datenschutz.org/standortdaten/ (30.04.2021).

DGfG (Deutsche Gesellschaft für Geographie) (2020): Bildungsstandards Geographie. DGfG, Berlin.

Dickel, M. (2016): Kartographische Konventionen über Kartenkunst befragen. Das Beispiel ,Alte Welt-Neue Welt' des Künstlers Stephan Huber. In: Gryl, I. (Hg.): Diercke Methoden. Reflexive Kartenarbeit. Westermann, Braunschweig. S. 208-221.

Dodge, M., R. Kitchin \& C. Perkins (Hrsg.) (2009): Rethinking Maps. New Frontiers in Cartographic Theory. Routledge, London.

Dorsch, C. \& D. Kanwischer (2019): Mündigkeitsorientierte Bildung in der geographischen Lehrkräftebildung. Zum Potential von E-Portfolios. In: Zeitschrift für Geographiedidaktik 47(3). S. 98-116. DOI: https://doi. org/10.18452/21264

Duggan, M. (2019): Cultures of Enthusiasm: An Ethnographic Study of Amateur Map-Maker Communities. In: Cartographica: The International Journal for Geographic Information and Geovisualization 54(3). S. 217-229. DOI: $10.3138 /$ cart.54.3.2018-0002

Edney, M. (1997): Mapping an Empire. The Geographical Construction of British India, 1765-1843. University of Chicago Press, Chicago.

Edney, M. (2019): Cartography. The ideal and its history. University of Chicago Press, Chicago.

Elden, S. (2007): Governmentality, Calculation, Territory. In: Environment and Planning D 25(3). S. 562-580. DOI: https://doi.org/10.1068/d428t

Foucault, M. (2000): Die Ordnung des Diskurses. Fischer, Frankfurt am Main.

Gryl, I. \& T. Jekel (2012): Re-centering geoinformation in secondary education: Toward a spatial citizenship approach. In: Cartographica 47(1). S. 18-28. DOI: https:// doi.org/10.3138/carto.47.1.18

Gryl, I. (2009): Kartenlesekompetenz. Ein Beitrag zum konstruktivistischen Geographieunterricht. Institut für Geographie und Regionalforschung, Wien.

Gryl, I. (2012): Geographielehrende, Reflexivität und Geomedien. Zur Konstruktion einer empirisch begründeten
Typologie. In: Geographie und ihre Didaktik (GuiD) 40(4). S. 161-182.

Gryl, I. (2016): Von der Orientierung zur Raumproduktion. GPS-Drawing und Mapping fördern geographisches Lernen in der Grundschule. In: Peschel, M. (Hg.): Mediales Lernen - Praxisbeispiele für eine Inklusive Mediendidaktik. Klinkhardt, Bad Heilbrunn. S. 53-63.

Gryl, I., D. Könen \& J. Pokraka (2017): Limits of freedom - defining a normative background for Spatial Citizenship. In: GI_Forum 2017(2). S. 3-12.

Habermas, J. (1970): Erkenntnis und Interesse. Suhrkamp, Berlin.

Halder, S. \& B. Michel (2018): Introduction - This is Not an Atlas. In: Orangotango+ (Hrsg.): This is not an Atlas. A Global Collection of Counter-Cartographies. transcript, Bielefeld. S. 12-21.

Hannah, M. (2000): Governmentality and the Mastery of Territory in Nineteenth-Century America. Cambridge University Press, Cambridge.

Harley, J. B. (1989): Deconstructing the Map. In: Cartographica 26(2). S. 1-20. DOI: https://doi.org/10.3138/ E635-7827-1757-9T53

Hemmer, M., I. Hemmer, A. Hüttermann \& M. Ulrich (2010): Kartenauswertungskompetenz. Theoretische Grundlagen und erste Überlegungen zu einem Kompetenzstrukturmodell. In: Geographie und ihre Didaktik (GuiD) 2010(3). S. 158-171.

Hintermann, C., F. Bergmeister, V. Kessel, H. Pichler \& D. Raithofer (2018): Hybride Identitäten im Kopf. Nachlese zur Abschlussveranstaltung des Sparkling Science Projekts MiDENTITY. In: GW-Unterricht 151. S. 59-62. DOI: https://doi.org/10.1553/gw-unterricht151s59

Jekel, T. (2008): In die Räume der GW-Didaktik. Briefe einer Reise. Materialien zur Didaktik der Geographie und Wirtschaftskunde 21. Institut für Geographie und Regionalforschung, Wien.

Kanwischer, D. \& A. Schlottmann (2017): Virale Raumkonstruktionen - Soziale Medien und Mündigkeit im Kontext gesellschaftswissenschaftlicher Medienbildung. In: Zeitschrift für Didaktik der Gesellschaftswissenschaften 8(2). S. 60-78.

Katz, C. (2021): Splanetary Urbanization. In: International Journal of Urban and Regional Research 45(4). S. 597611. DOI: https://doi.org/10.1111/1468-2427.13025.

Lehner, M., J. Pokraka \& I. Gryl (2019): From 'the map' to an internalized concept. Developing a method of deconstruction as practice for reflexive cartography. In: GI_ Forum 1. S. 194-205. DOI: https://doi.org/10.1553/ giscience2019_02_s194

MacEachren, A. M. (1994): Visualization in modern cartography: setting the agenda. In: Taylor, D. \& A. M. MacEachren (Hrsg.): Visualization in modern cartography. Pergamon, Headington. S. 1-12.

Michel, B. (2017): Forensische Blicke und Praktiken kritischer Geovisualisierung. In: ACME: An International E-Journal for Critical Geographies 16. S. 687-712. 
Michel, B. (2021): Kartographische Raumproduktionen und Kritische Kartographien. In: Kogler, R. \& J. Wintzer (Hrsg.): Raum und Bild - Strategien visueller raumbezogener Forschung. Springer, Berlin. S. 143-155.

Mogel, L. \& A. Bhagat (Hrsg.) (2008): An Atlas of Radical Cartography. Journal of Aesthetics and Protest Press, Los Angeles.

Nöthen, E. (2016): Aesthetic Mapping - Kartographische Reflexion von ästhetischen Erfahrungen während des Reisens. In: Gryl, I. (Hg.): Diercke - Reflexive Kartenarbeit. Methoden und Aufgaben. Westermann, Braunschweig. S. 201-207.

O'Rourke, K. (2016): Walking and mapping. Artists as cartographers. The MIT Press, Cambridge, MA.

Orangotango+ (Hrsg.) (2018): This is not an Atlas. A Global Collection of Counter-Cartographies. Transcript, Bielefeld. https://www.transcript-verlag.de/media/pdf/ c2/9f/76/oa9783839445198GVHWZBYklUKNq.pdf (15.09.2021)

Paasi, A. (1986): The institutionalization of regions. A theoretical framework for understanding the emergence of regions and the constitution of regional identity. In: Fennia 1986(1). S. 105-146.

Pettig, F. (2019): Kartographische Streifzüge. Ein Baustein zur phänomenologischen Grundlegung der Geographiedidaktik. Transcript, Bielefeld.

Pickles, J. (2004): A History of Space. Cartographic Reason, Mapping and the Geo-coded World. Routledge, London.

Rinschede, G. (2003): Geographiedidaktik. Schöningh, Paderborn.

Sauer, C. (1956): The Education of a Geographer. In: Annals of the Association of American Geographers 46(3). S. 287-299.

Scott, J. C. (1998): Seeing like a state. How certain schemes to improve the human condition have failed. Yale University Press, New Haven.

Sletto, B., A. Almeida, B. de Wagner, J. Bryan, \& C. R. Hale (Hrsg.) (2020): Radical cartographies. Participatory mapmaking from Latin America. First Ed. University of Texas Press, Austin.

Stalder, F. (2017): Kultur der Digitalität. Suhrkamp, Berlin.

Stark, H. J. (2012): Spatial pattern analysis of secondary school students' leisure tracks. In: Car, A. et al. (Hrsg.): GI_Forum 2012. Wichmann, Berlin. S. 336-344.

The Guardian (2017): Fitness tracking app Strava gives away location of secret US army bases. https://www.theguardian.com/world/2018/jan/28/fitness-tracking-app-givesaway-location-of-secret-us-army-bases (30.04.2021).

Turnbull, D. (1998): Mapping encounters and (en)countering maps: A critical examination of cartographic resistance. In: Knowledge and Society 11. S. 15-44.

UN (United Nations) (2006): Convention on the Rights of Persons with Disabilities. https://www.un.org/development/desa/disabilities/convention-on-the-rights-ofpersons-with-disabilities/convention-on-the-rights-ofpersons-with-disabilities-2.html (30.04.2021).

Vielhaber, C. (2000): Vermittlung und Interesse. Zwei Schlüsselkategorien fachdidaktischer Grundlegungen im Geographieunterricht. In: Vielhaber, C. (Hg.): Geographiedidaktik kreuz und quer. Vom Vermittlungsinteresse bis zum Methodenstreit. Institut für Geographie und Regionalforschung Wien, Wien. S. 9-26.

Warren, G., C. Katz \& N. Heynen (2019): Myths, Cults, Memories, and Revisions in Radical Geographic History. Revisiting the Detroit Geographical Expedition and Institute. In: Barnes, T. \& E. Sheppard (Hrsg.): Spatial histories of radical geography. North America and beyond. John Wiley \& Sons Ltd, Hoboken. S. 59-85.

Werlen, B. (1993): Society action and space: An alternative human geography. Routledge, London.

Wheelmap.org (o. J.): Mitmachen. https://news.wheelmap. org/mitmachen/ (29.04.2021).

Wood, D. (1992): The Power of Maps. Guilford, New York.

Wood, D. (2010): Rethinking the Power of Maps. Guilford, New York. 